\title{
A New Kinetically Preferable Polymorph of 1-(4'- Cyanobenzyl)pyridinium bis(maleonitriledithiolato)nickelate with Spin-Peierls-Type Transition
}

Guo-Jun Yuan, ${ }^{\mathrm{a}, \mathrm{b}}$ Dong-Sheng Shao, ${ }^{\mathrm{a}}$ Qiu Ren, ${ }^{\mathrm{a}}$ Fei-Yan Feng, ${ }^{\mathrm{b}}$ Hao Yang, ${ }^{\mathrm{a}}$ Lifeng Wang, ${ }^{c}$ Xiao-Ming Ren*a

a State Key Laboratory of Materials-Oriented Chemical Engineering and College of Chemistry and Molecular Engineering, Nanjing Tech University, Nanjing 211816, P. R. China

${ }^{\mathrm{b}}$ Key Laboratory of Advanced Materials of Nanjing and Department of Chemistry, Nanjing Xiaozhuang University, Nanjing 211171, P. R. China

${ }^{\mathrm{c}}$ Institute for Frontier Materials (IFM), Deakin University, 75 Pigdons Road, Waurn Ponds, Victoria 3216, Australia

Tel.: +86 2558139476

Email: xmren@njtech.edu.cn 


\section{Contents}

Figure S1: Comparison of experimental and simulated Powder X-ray diffraction profiles for $\alpha-[\mathrm{CN}-\mathrm{Py}]\left[\mathrm{Ni}(\mathrm{mnt})_{2}\right]$ and $\beta-[\mathrm{CN}-\mathrm{Py}]\left[\mathrm{Ni}(\mathrm{mnt})_{2}\right]$ at room temperature in HTP.

Figure S2: Illustration of the adjacent anions in an anion stack and the adjacent cations in a cation stack related via symmetry transformation in HTP of $\beta-[\mathrm{CN}-\mathrm{Py}]\left[\mathrm{Ni}(\mathrm{mnt})_{2}\right]$. (a) $\mathrm{Ni}(1)$ atom locates at a general site with the fraction coordinate of $(\mathrm{x}, \mathrm{y}, \mathrm{z})$, its two neighbors $\mathrm{Ni}(1) \# 1$ and $\mathrm{Ni}(1) \# 2$ atoms in an anion stack are generated via the symmetric transforms $\# 1=\mathrm{x}, 0.5-\mathrm{y},-0.5+\mathrm{z}$ and $\# 2=\mathrm{x}, 0.5-\mathrm{y}$, $0.5+\mathrm{z}$ with the corresponding fraction coordinates $(\mathrm{x}, 0.5-\mathrm{y},-0.5+\mathrm{z})$ and $(\mathrm{x}, 0.5-\mathrm{y}$, $0.5+z$ ), respectively. (b) If the Center 1 of the phenyl ring has the fraction coordinate of $(\mathrm{x}, \mathrm{y}, \mathrm{z})$, its two neighbors Center $1 \# 1$ and Center $1 \# 2$ have the fraction coordinates of $(\mathrm{x}, 0.5-\mathrm{y},-0.5+\mathrm{z})$ and $(\mathrm{x}, 0.5-\mathrm{y}, 0.5+\mathrm{z})$, respectively.

Figure S3: $(\mathrm{a}, \mathrm{b})$ 1-D regular $\left[\mathrm{Ni}(\mathrm{mnt})_{2}\right]^{-}$chain $(\mathrm{c}, \mathrm{d})$ regular cation stack for $\alpha$-[CN-Py][Ni(mnt) $\left.)_{2}\right]$ in both HTP and LTP.

Figure S4: (a) Charge-assisted H-bond interactions between anion and cation stacks as well as between the neighboring cation stacks (b) the relative orientation of 2-D supramolecular H-bond network and the crystallographic ( $\left(\begin{array}{lll}1 & 0 & 0\end{array}\right)$ plane in $\alpha-[\mathrm{CN}-\mathrm{Py}]\left[\mathrm{Ni}(\mathrm{mnt})_{2}\right]$.

Figure S5: DSC curve of $\alpha-[\mathrm{CN}-\mathrm{Py}]\left[\mathrm{Ni}(\mathrm{mnt})_{2}\right]$.

Figure S6: Variable temperature AC conductivity of $\beta-[\mathrm{CN}-\mathrm{Py}]\left[\mathrm{Ni}(\mathrm{mnt})_{2}\right]$ in the temperature ranges of $123-373 \mathrm{~K}$ at the selected frequencies, which show rather low conduction. Most importantly, no sizable change of conductivity at the magnetic phase transition critical temperature, and this observation demonstrates the transition is magnetic but not electronic phase transition. As a result, this is 1D spin-Peierls transition system but not Peierls transition system.

Table S1: Crystallographic data and refinement parameters for $\alpha-[\mathrm{CN}-\mathrm{Py}]\left[\mathrm{Ni}(\mathrm{mnt})_{2}\right]$ in HTP in the literature and this work

Table S2: Crystallographic data and refinement parameters for $\alpha-[\mathrm{CN}-\mathrm{Py}]\left[\mathrm{Ni}(\mathrm{mnt})_{2}\right]$ in LTP at room temperature in the literature 
Table S3: Comparison of bond lengths in the moieties of anion and cation between single crystal at $296 \mathrm{~K}$ and geometry optimization in $\alpha-[\mathrm{CN}-\mathrm{Py}]\left[\mathrm{Ni}(\mathrm{mnt})_{2}\right]$ Table S4: Comparison of bond angles in the moieties of anion and cation between single crystal at $296 \mathrm{~K}$ and geometry optimization in $\alpha-[\mathrm{CN}-\mathrm{Py}]\left[\mathrm{Ni}(\mathrm{mnt})_{2}\right]$ Table S5: Comparison of bond lengths in the moieties of anion and cation between single crystal at $296 \mathrm{~K}$ and geometry optimization in $\beta-[\mathrm{CN}-\mathrm{Py}]\left[\mathrm{Ni}(\mathrm{mnt})_{2}\right]$ Table S6: Comparison of bond angles in the moieties of anion and cation between single crystal at $296 \mathrm{~K}$ and geometry optimization in $\beta-[\mathrm{CN}-\mathrm{Py}]\left[\mathrm{Ni}(\mathrm{mnt})_{2}\right]$

\section{References}

1. Xie, J. L.; Ren, X. M.; He, C.; Song, Y.; Meng, Q. J.; Kremer, R. K.; Yao, Y. G. Chem. Phys. Lett. 2003, 369, 41-48. 

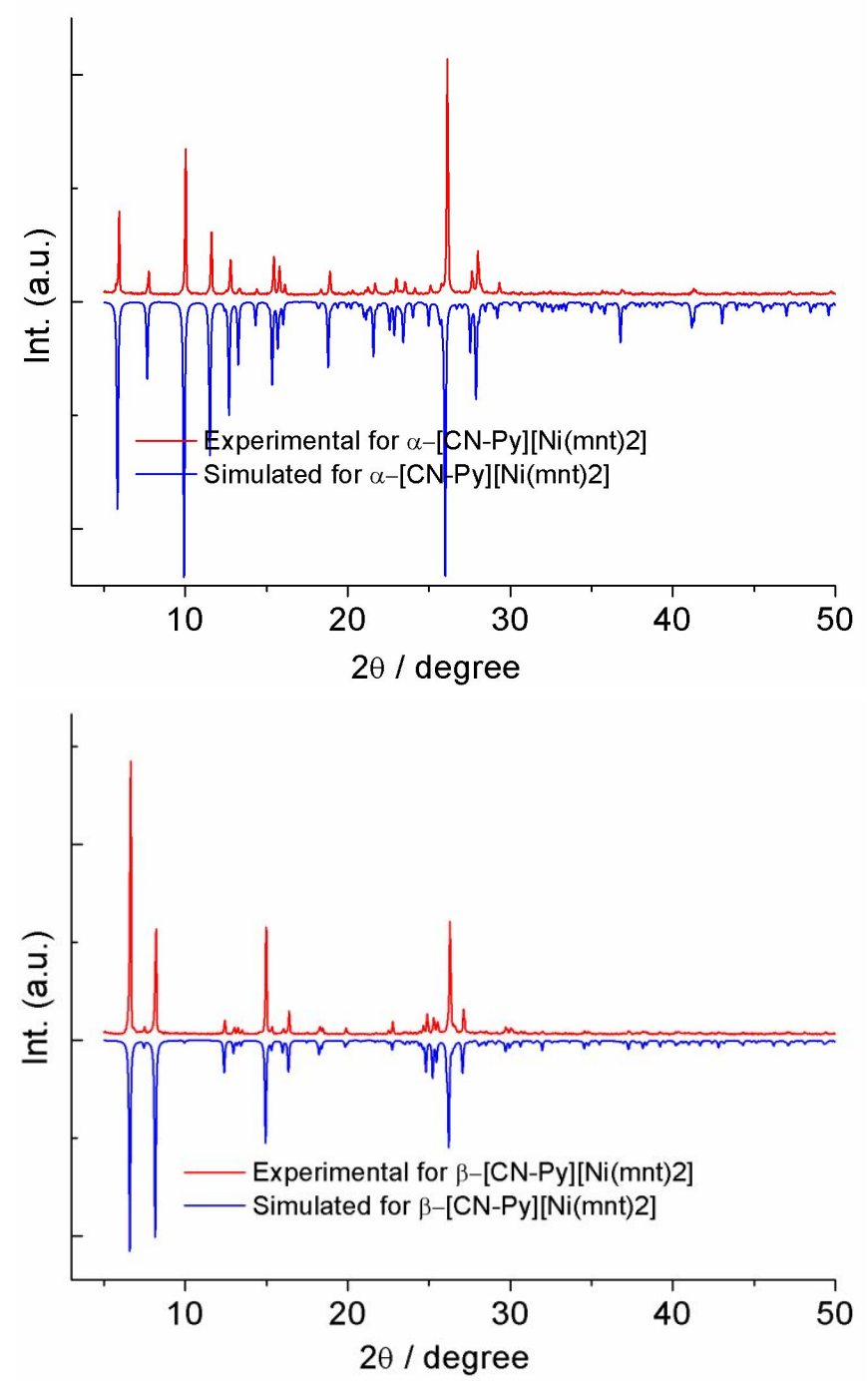

Figure S1: Comparison of experimental and simulated Powder X-ray diffraction profiles for $\alpha-[\mathrm{CN}-\mathrm{Py}]\left[\mathrm{Ni}(\mathrm{mnt})_{2}\right]$ and $\beta-[\mathrm{CN}-\mathrm{Py}]\left[\mathrm{Ni}(\mathrm{mnt})_{2}\right]$ at room temperature in HTP. 
(a)

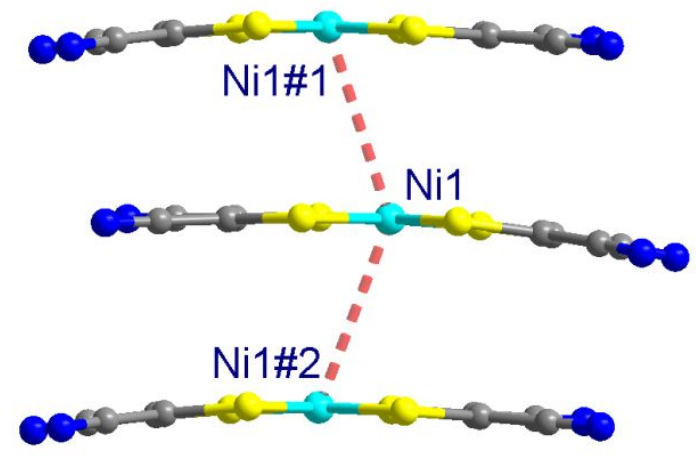

(b)

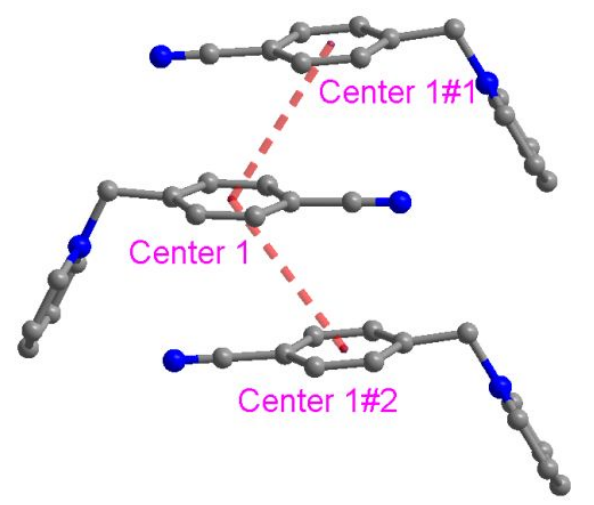

Figure S2: Illustration of the adjacent anions in an anion stack and the adjacent cations in a cation stack related via symmetry transformation in HTP of $\beta$-[CN-Py] $\left[\mathrm{Ni}(\mathrm{mnt})_{2}\right]$. (a) $\mathrm{Ni}(1)$ atom locates at a general site with the fraction coordinate of $(\mathrm{x}, \mathrm{y}, \mathrm{z})$, its two neighbors $\mathrm{Ni}(1) \# 1$ and $\mathrm{Ni}(1) \# 2$ atoms in an anion stack are generated via the symmetric transforms $\# 1=\mathrm{x}, 0.5-\mathrm{y},-0.5+\mathrm{z}$ and $\# 2=\mathrm{x}, 0.5-\mathrm{y}$, $0.5+\mathrm{z}$ with the corresponding fraction coordinates $(\mathrm{x}, 0.5-\mathrm{y},-0.5+\mathrm{z})$ and $(\mathrm{x}, 0.5-\mathrm{y}$, $0.5+\mathrm{z}$ ), respectively. (b) If the Center 1 of the phenyl ring has the fraction coordinate of $(\mathrm{x}, \mathrm{y}, \mathrm{z})$, its two neighbors Center $1 \# 1$ and Center $1 \# 2$ have the fraction coordinates of $(\mathrm{x}, 0.5-\mathrm{y},-0.5+\mathrm{z})$ and $(\mathrm{x}, 0.5-\mathrm{y}, 0.5+\mathrm{z})$, respectively. 
(a)

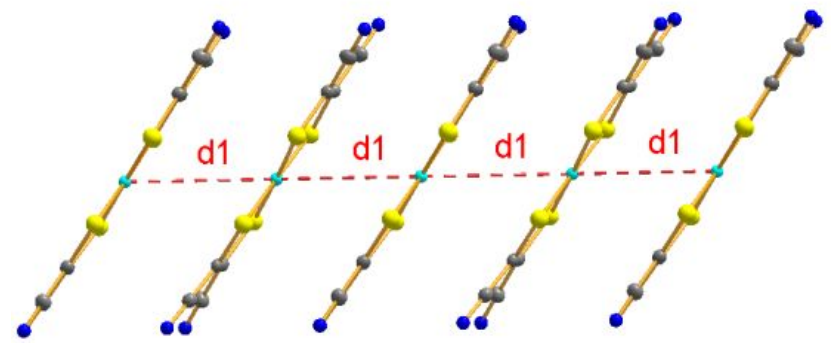

(b)

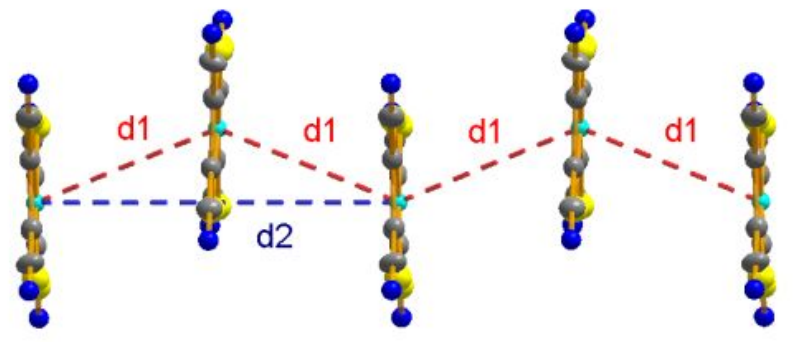

(c)

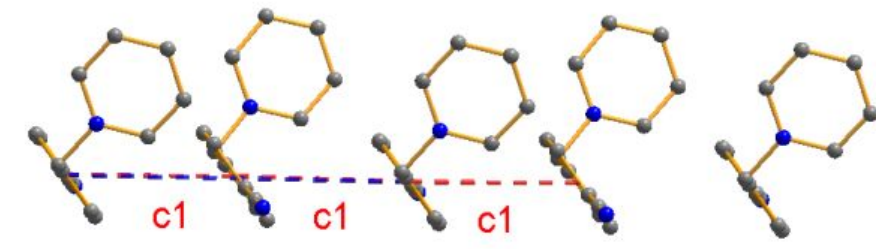

(d)

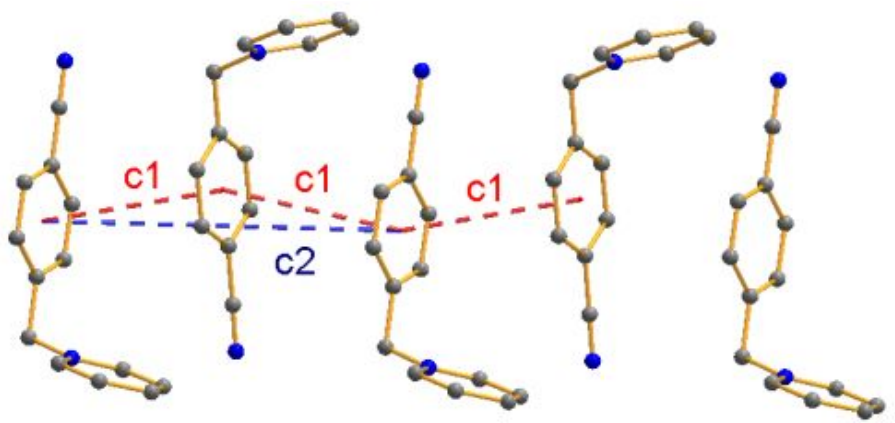

Figure S3: $(\mathrm{a}, \mathrm{b})$ 1-D regular $\left[\mathrm{Ni}(\mathrm{mnt})_{2}\right]^{-}$chain $(\mathrm{c}, \mathrm{d})$ regular cation stack for $\alpha-[\mathrm{CN}-\mathrm{Py}]\left[\mathrm{Ni}(\mathrm{mnt})_{2}\right]$ in both HTP and LTP. 


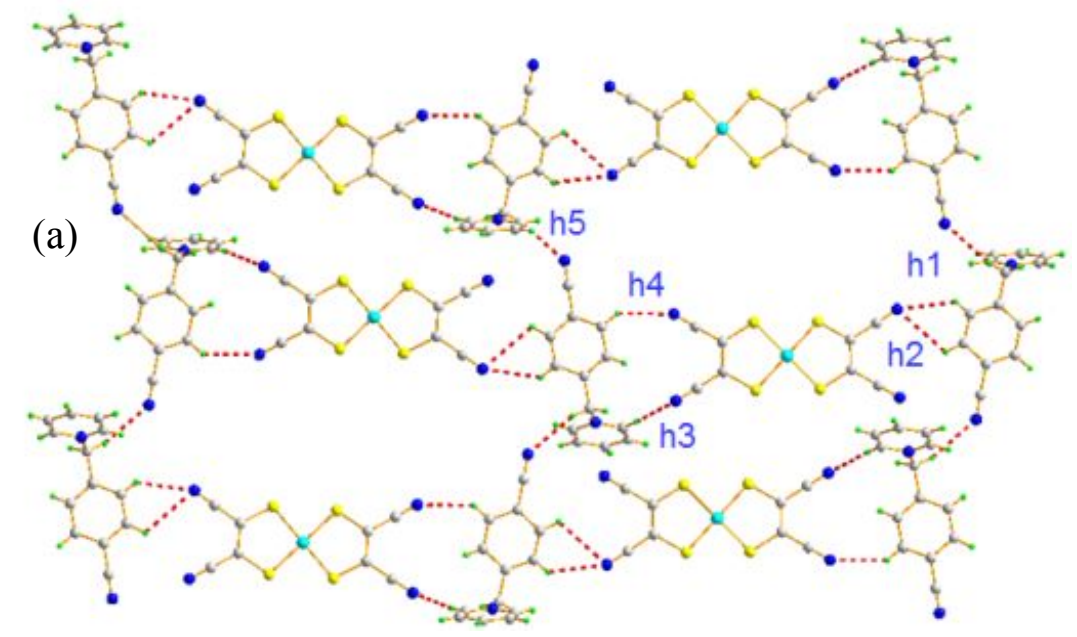

(b)

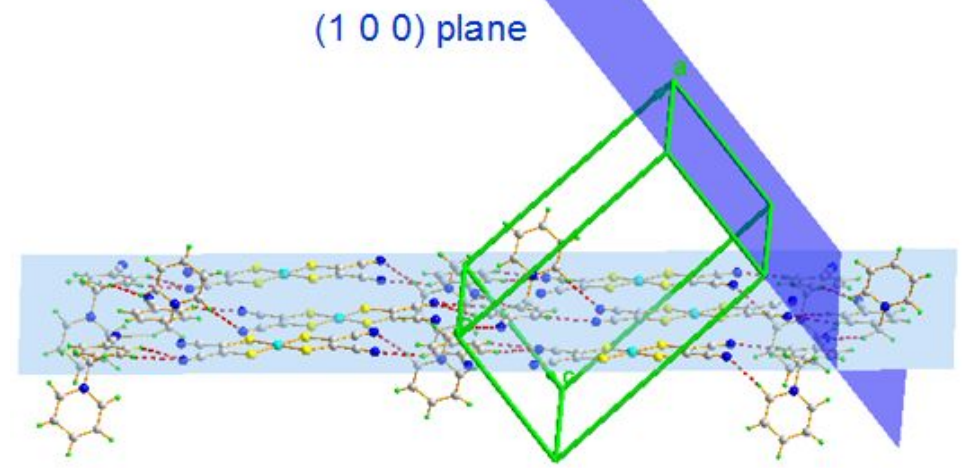

Figure S4: (a) Charge-assisted H-bond interactions between anion and cation stacks as well as between the neighboring cation stacks (b) the relative orientation of 2-D supramolecular H-bond network and the crystallographic (lll 10 plane in $\alpha-[\mathrm{CN}-\mathrm{Py}]\left[\mathrm{Ni}(\mathrm{mnt})_{2}\right]$.

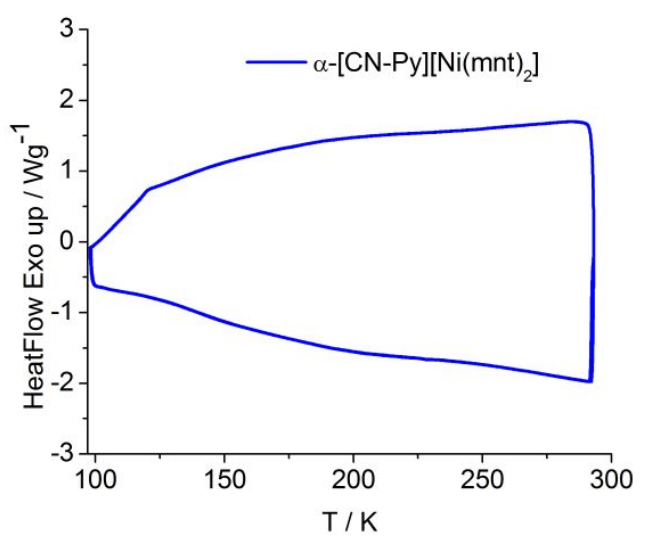

Figure S5: DSC curve of $\alpha-[\mathrm{CN}-\mathrm{Py}]\left[\mathrm{Ni}(\mathrm{mnt})_{2}\right]$. 

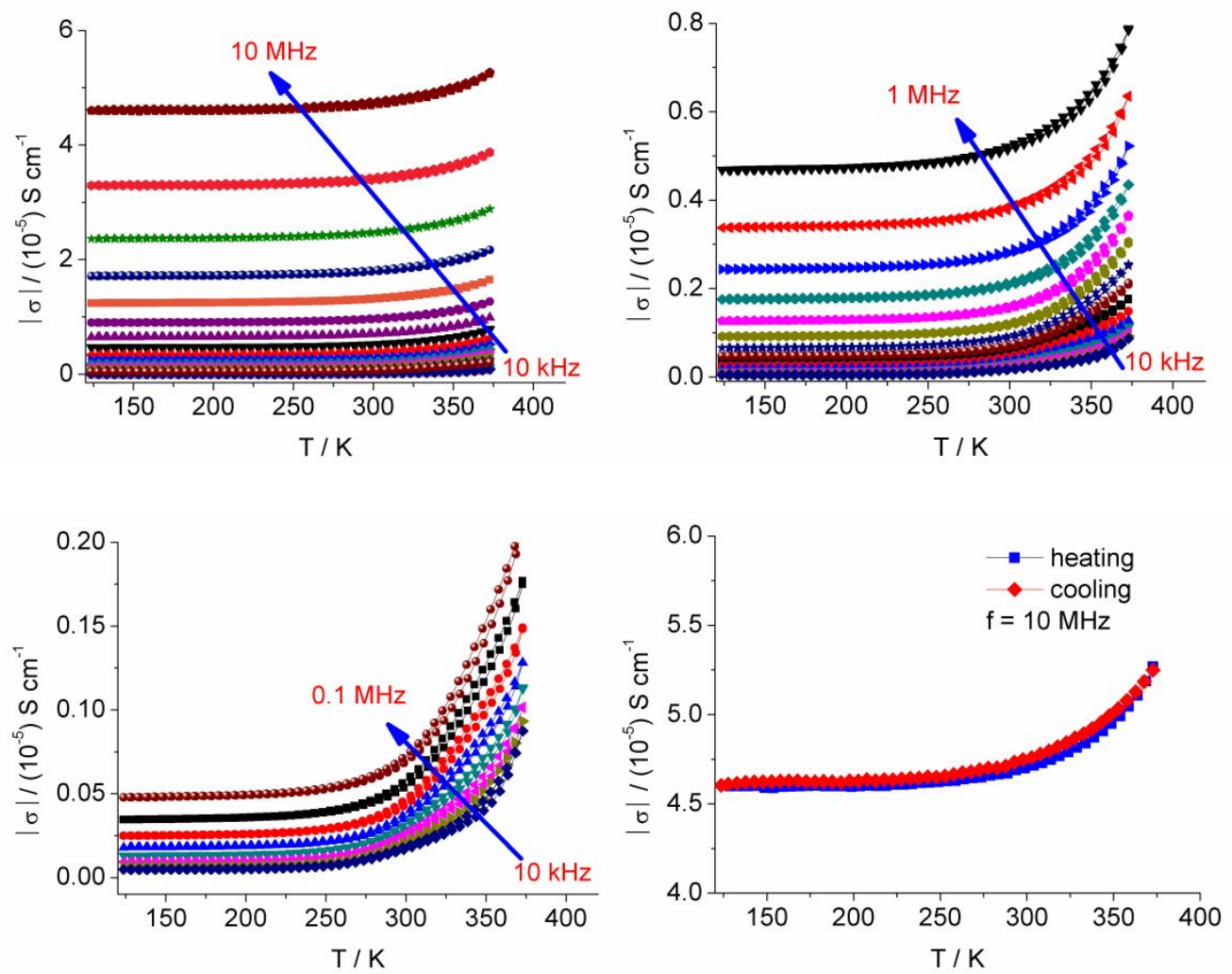

Figure S6: Variable temperature $\mathrm{AC}$ conductivity of $\beta-[\mathrm{CN}-\mathrm{Py}]\left[\mathrm{Ni}(\mathrm{mnt})_{2}\right]$ in the temperature ranges of $123-373 \mathrm{~K}$ at the selected frequencies, which show rather low conduction. Most importantly, no sizable change of conductivity at the magnetic phase transition critical temperature, and this observation demonstrates the transition is magnetic but not electronic phase transition. As a result, this is 1D spin-Peierls transition system but not Peierls transition system. 
Table S1: Crystallographic data and refinement parameters for $\alpha-[\mathrm{CN}-\mathrm{Py}]\left[\mathrm{Ni}(\mathrm{mnt})_{2}\right]$ in HTP in the literature and this work

\begin{tabular}{|c|c|c|}
\hline & This work & Ref. 1 \\
\hline Temp. / K & $296(2)$ & 293(2) \\
\hline Wavelength / $\AA$ & 0.71073 & 0.71073 \\
\hline Formula & $\mathrm{C}_{21} \mathrm{H}_{11} \mathrm{~N}_{6} \mathrm{NiS}_{4}$ & $\mathrm{C}_{21} \mathrm{H}_{11} \mathrm{~N}_{6} \mathrm{NiS}_{4}$ \\
\hline Formula weight & 534.31 & 534.31 \\
\hline Space group & $P 2{ }_{l} / c$ & $P 2{ }_{1} / c$ \\
\hline CCDC no. & 932830 & 182195 \\
\hline Crystal system & Monoclinic & Monoclinic \\
\hline$a / \AA$ & $15.206(3)$ & $15.197(3)$ \\
\hline$b / \AA$ & $17.803(3)$ & $17.796(4)$ \\
\hline$c / \AA$ & $8.6171(16)$ & $8.6089(17)$ \\
\hline$\beta /^{\circ}$ & $95.156(3)$ & $95.15(3)$ \\
\hline$V / \AA^{3} / \mathrm{Z}$ & $2323.4(7) / 4$ & $2318.9(8) / 4$ \\
\hline$\rho / \mathrm{g} \cdot \mathrm{cm}^{-1}$ & 1.527 & 1.530 \\
\hline$F(000)$ & 1084 & 1084 \\
\hline Abs.coeff. $/ \mathrm{mm}^{-1}$ & 1.216 & 1.219 \\
\hline$\theta$ Range for data collection $\left(^{\circ}\right)$ & $1.34-25.59$ & $3.48-27.49$ \\
\hline & $-18 \leq \mathrm{h} \leq 18$ & $-19 \leq \mathrm{h} \leq 19$ \\
\hline Index ranges & $-21 \leq \mathrm{k} \leq 18$ & $-23 \leq \mathrm{k} \leq 23$ \\
\hline & $-10 \leq 1 \leq 8$ & $-11 \leq 1 \leq 11$ \\
\hline $\mathrm{R}_{\text {int }}$ & 0.0848 & 0.0427 \\
\hline $\begin{array}{l}\text { Independent reflect. } \\
\text { /restraints/parameters }\end{array}$ & $4317 / 0 / 290$ & $5303 / 0 / 289$ \\
\hline Refinement method & \multicolumn{2}{|c|}{ The least square refinement on $\mathrm{F}^{2}$} \\
\hline Goodness of fit on $F^{2}$ & 0.865 & 1.024 \\
\hline \multirow{2}{*}{$R_{1}, w R_{2}[I>2 \sigma(\mathrm{I})]$} & $R_{1}=0.0502$ & $R_{1}=0.0332$ \\
\hline & $w R_{2}=0.0873$ & $w R_{2}=0.0733$ \\
\hline \multirow{2}{*}{$R_{1}, w R_{2}$ [all data] } & $R_{1}=0.1473$ & $R_{1}=0.0582$ \\
\hline & $w R_{2}=0.1085$ & $w R_{2}=0.0835$ \\
\hline Residual / e $\cdot \AA^{-3}$ & $0.460 /-0.313$ & $0.253 /-0.342$ \\
\hline
\end{tabular}


Table S2: Crystallographic data and refinement parameters for $\alpha-[\mathrm{CN}-\mathrm{Py}]\left[\mathrm{Ni}(\mathrm{mnt})_{2}\right]$ in LTP at room temperature in the literature

\begin{tabular}{|c|c|c|}
\hline & Ref. 1 & Ref. 1 \\
\hline Temp. / K & $180(2)$ & $140(2)$ \\
\hline Wavelength / $\AA$ & 0.71073 & 0.71073 \\
\hline Formula & $\mathrm{C}_{21} \mathrm{H}_{11} \mathrm{~N}_{6} \mathrm{NiS}_{4}$ & $\mathrm{C}_{21} \mathrm{H}_{11} \mathrm{~N}_{6} \mathrm{NiS}_{4}$ \\
\hline Formula weight & 534.31 & 534.31 \\
\hline Space group & $P 2_{1} / c$ & $P 2_{1} / c$ \\
\hline CCDC no. & 186340 & 186341 \\
\hline Crystal system & Monoclinic & Monoclinic \\
\hline$a / \AA$ & $15.0630(3)$ & $15.0218(3)$ \\
\hline$b / \AA$ & $17.7252(4)$ & $17.6926(3)$ \\
\hline$c / \AA$ & $8.5663(2)$ & $8.54580(10)$ \\
\hline$\alpha /^{\circ}$ & 90 & 90 \\
\hline$\beta / \circ$ & $94.6499(10)$ & $94.5089(8)$ \\
\hline$\gamma /{ }^{\circ}$ & 90 & 90 \\
\hline$V / \AA^{3} / Z$ & $2279.63(9) / 4$ & $2264.23(6) / 4$ \\
\hline$\rho / \mathrm{g} \cdot \mathrm{cm}^{-1}$ & 1.557 & 1.567 \\
\hline$F(000)$ & 1084 & 1084 \\
\hline Abs.coeff. / mm ${ }^{-1}$ & 1.239 & 1.248 \\
\hline$\theta$ Range for data collection $\left(^{\circ}\right)$ & $3.51-27.48$ & $3.52-27.48$ \\
\hline & $-19 \leq \mathrm{h} \leq 19$ & $-19 \leq \mathrm{h} \leq 19$ \\
\hline Index ranges & $-22 \leq \mathrm{k} \leq 22$ & $-22 \leq \mathrm{k} \leq 22$ \\
\hline & $-11 \leq 1 \leq 11$ & $-11 \leq 1 \leq 11$ \\
\hline $\mathrm{R}_{\text {int }}$ & 0.0928 & 0.0503 \\
\hline $\begin{array}{ll}\text { Independent } & \text { reflect. } \\
\text { /restraints/parameters } & \end{array}$ & $5099 / 0 / 289$ & $5130 / 0 / 289$ \\
\hline Refinement method & \multicolumn{2}{|c|}{ The least square refinement on $\mathrm{F}^{2}$} \\
\hline Goodness of fit on $F^{2}$ & 1.008 & 1.031 \\
\hline \multirow{2}{*}{$R_{1}, w R_{2}[I>2 \sigma(\mathrm{I})]$} & $R_{1}=0.0386$ & $R_{1}=0.0283$ \\
\hline & $w R_{2}=0.0693$ & $w R_{2}=0.0594$ \\
\hline \multirow{2}{*}{$R_{1}, w R_{2}$ [all data] } & $R_{1}=0.1012$ & $R_{1}=0.0511$ \\
\hline & $w R_{2}=0.0823$ & $w R_{2}=0.0652$ \\
\hline Residual / e $\cdot \AA^{-3}$ & $0.317 /-0.398$ & $0.269 /-0.298$ \\
\hline
\end{tabular}


Table S3: Comparison of bond lengths in the moieties of anion and cation between single crystal at $296 \mathrm{~K}$ and geometry optimization in $\alpha-[\mathrm{CN}-\mathrm{Py}]\left[\mathrm{Ni}(\mathrm{mnt})_{2}\right]$

\begin{tabular}{|c|c|c|}
\hline \multirow{2}{*}{ Atom pair } & \multicolumn{2}{|c|}{ Bond distance / $\AA$} \\
\hline & Single crystal daata & Geometry optimization \\
\hline $\mathrm{Ni} \ldots \mathrm{S} 4$ & $2.1387(15)$ & 2.141 \\
\hline $\mathrm{Ni} \ldots \mathrm{S} 3$ & $2.1487(16)$ & 2.151 \\
\hline $\mathrm{Ni}$...S1 & $2.1493(16)$ & 2.147 \\
\hline $\mathrm{Ni} \ldots \mathrm{S} 2$ & $2.1509(15)$ & 2.151 \\
\hline $\mathrm{S} 1 \ldots \mathrm{C} 2$ & $1.713(5)$ & 1.715 \\
\hline $\mathrm{S} 2 \ldots \mathrm{C} 3$ & $1.708(5)$ & 1.716 \\
\hline S3 ...C6 & $1.714(5)$ & 1.716 \\
\hline $\mathrm{S} 4 \ldots \mathrm{C} 7$ & $1.707(5)$ & 1.716 \\
\hline $\mathrm{N} 1 \ldots \mathrm{C} 1$ & $1.152(6)$ & 1.185 \\
\hline $\mathrm{N} 2 \ldots \mathrm{C} 4$ & $1.142(6)$ & 1.184 \\
\hline $\mathrm{N} 3 \ldots \mathrm{C} 5$ & $1.129(6)$ & 1.185 \\
\hline $\mathrm{N} 4 \ldots \mathrm{C} 8$ & $1.136(6)$ & 1.185 \\
\hline $\mathrm{N} 5 \ldots \mathrm{C} 21$ & $1.142(6)$ & 1.183 \\
\hline N6...C13 & $1.327(7)$ & 1.355 \\
\hline N6...C9 & $1.339(7)$ & 1.355 \\
\hline N6...C14 & $1.487(7)$ & 1.490 \\
\hline $\mathrm{C} 1 \ldots \mathrm{C} 2$ & $1.429(7)$ & 1.408 \\
\hline $\mathrm{C} 2 \ldots \mathrm{C} 3$ & $1.365(6)$ & 1.393 \\
\hline $\mathrm{C} 3 \ldots \mathrm{C} 4$ & $1.433(7)$ & 1.409 \\
\hline $\mathrm{C} 5 \ldots \mathrm{C} 6$ & $1.442(7)$ & 1.410 \\
\hline C6...C7 & $1.369(6)$ & 1.392 \\
\hline $\mathrm{C} 7 \ldots \mathrm{C} 8$ & $1.442(7)$ & 1.410 \\
\hline C9...C10 & $1.367(9)$ & 1.382 \\
\hline $\mathrm{C} 10 \ldots \mathrm{C} 11$ & $1.369(10)$ & 1.392 \\
\hline $\mathrm{C} 11 \ldots \mathrm{C} 12$ & $1.351(10)$ & 1.392 \\
\hline $\mathrm{C} 12 \ldots \mathrm{C} 13$ & $1.359(9)$ & 1.381 \\
\hline $\mathrm{C} 14 \ldots \mathrm{C} 15$ & $1.516(7)$ & 1.508 \\
\hline $\mathrm{C} 15 \ldots \mathrm{C} 16$ & $1.375(7)$ & 1.398 \\
\hline C16...C17 & $1.386(7)$ & 1.388 \\
\hline $\mathrm{C} 17 \ldots \mathrm{C} 18$ & $1.380(7)$ & 1.404 \\
\hline C18...C19 & $1.389(7)$ & 1.403 \\
\hline $\mathrm{C} 19 \ldots \mathrm{C} 20$ & $1.366(8)$ & 1.388 \\
\hline
\end{tabular}


Table S4: Comparison of bond angles in the moieties of anion and cation between single crystal at $296 \mathrm{~K}$ and geometry optimization in $\alpha-[\mathrm{CN}-\mathrm{Py}]\left[\mathrm{Ni}(\mathrm{mnt})_{2}\right]$

\begin{tabular}{|c|c|c|}
\hline \multirow{2}{*}{$\angle$ atom1-atom2-atom3 } & \multicolumn{2}{|c|}{ Bond angles $/^{\circ}$} \\
\hline & Single crystal daata & Geometry optimization \\
\hline $\mathrm{S}(4)-\mathrm{Ni}(1)-\mathrm{S}(3)$ & $92.49(6)$ & 92.276 \\
\hline $\mathrm{S}(4)-\mathrm{Ni}(1)-\mathrm{S}(1)$ & $178.13(7)$ & 173.900 \\
\hline $\mathrm{S}(3)-\mathrm{Ni}(1)-\mathrm{S}(1)$ & $87.84(6)$ & 88.135 \\
\hline $\mathrm{S}(4)-\mathrm{Ni}(1)-\mathrm{S}(2)$ & $87.22(6)$ & 87.651 \\
\hline $\mathrm{S}(3)-\mathrm{Ni}(1)-\mathrm{S}(2)$ & $179.40(7)$ & 175.199 \\
\hline $\mathrm{S}(1)-\mathrm{Ni}(1)-\mathrm{S}(2)$ & $92.47(6)$ & 92.447 \\
\hline $\mathrm{C}(2)-\mathrm{S}(1)-\mathrm{Ni}(1)$ & $103.18(18)$ & 103.941 \\
\hline $\mathrm{C}(3)-\mathrm{S}(2)-\mathrm{Ni}(1)$ & $103.2(2)$ & 103.782 \\
\hline $\mathrm{C}(6)-\mathrm{S}(3)-\mathrm{Ni}(1)$ & $103.15(19)$ & 103.843 \\
\hline $\mathrm{C}(7)-\mathrm{S}(4)-\mathrm{Ni}(1)$ & $103.68(19)$ & 104.327 \\
\hline $\mathrm{C}(13)-\mathrm{N}(6)-\mathrm{C}(9)$ & $119.8(6)$ & 121.176 \\
\hline $\mathrm{C}(13)-\mathrm{N}(6)-\mathrm{C}(14)$ & $120.0(6)$ & 118.970 \\
\hline $\mathrm{C}(9)-\mathrm{N}(6)-\mathrm{C}(14)$ & $120.2(6)$ & 119.837 \\
\hline $\mathrm{N}(1)-\mathrm{C}(1)-\mathrm{C}(2)$ & $178.8(7)$ & 178.547 \\
\hline$C(3)-C(2)-C(1)$ & $121.2(5)$ & 121.483 \\
\hline $\mathrm{C}(3)-\mathrm{C}(2)-\mathrm{S}(1)$ & $120.4(5)$ & 119.901 \\
\hline$C(1)-C(2)-S(1)$ & $118.4(4)$ & 118.608 \\
\hline $\mathrm{C}(2)-\mathrm{C}(3)-\mathrm{C}(4)$ & $120.5(5)$ & 121.538 \\
\hline$C(2)-C(3)-S(2)$ & $120.7(5)$ & 119.894 \\
\hline $\mathrm{C}(4)-\mathrm{C}(3)-\mathrm{S}(2)$ & $118.8(4)$ & 118.549 \\
\hline $\mathrm{N}(2)-\mathrm{C}(4)-\mathrm{C}(3)$ & $178.9(6)$ & 179.125 \\
\hline $\mathrm{N}(3)-\mathrm{C}(5)-\mathrm{C}(6)$ & $179.8(8)$ & 178.471 \\
\hline$C(7)-C(6)-C(5)$ & $121.3(5)$ & 121.201 \\
\hline$C(7)-C(6)-S(3)$ & $120.4(5)$ & 119.958 \\
\hline $\mathrm{C}(5)-\mathrm{C}(6)-\mathrm{S}(3)$ & $118.3(4)$ & 118.832 \\
\hline$C(6)-C(7)-C(8)$ & $121.1(5)$ & 122.147 \\
\hline$C(6)-C(7)-S(4)$ & $120.3(5)$ & 119.522 \\
\hline $\mathrm{C}(8)-\mathrm{C}(7)-\mathrm{S}(4)$ & $118.6(4)$ & 118.322 \\
\hline $\mathrm{N}(4)-\mathrm{C}(8)-\mathrm{C}(7)$ & 179.6(7) & 178.787 \\
\hline $\mathrm{N}(6)-\mathrm{C}(9)-\mathrm{C}(10)$ & $120.5(7)$ & 120.176 \\
\hline$C(9)-C(10)-C(11)$ & $119.0(8)$ & 119.514 \\
\hline$C(12)-C(11)-C(10)$ & $120.0(8)$ & 119.348 \\
\hline$C(11)-C(12)-C(13)$ & $118.9(8)$ & 119.341 \\
\hline $\mathrm{N}(6)-\mathrm{C}(13)-\mathrm{C}(12)$ & $121.7(7)$ & 120.411 \\
\hline $\mathrm{N}(6)-\mathrm{C}(14)-\mathrm{C}(15)$ & $110.1(5)$ & 111.216 \\
\hline$C(16)-C(15)-C(20)$ & $118.8(6)$ & 119.629 \\
\hline$C(16)-C(15)-C(14)$ & $121.0(6)$ & 120.284 \\
\hline$C(20)-C(15)-C(14)$ & $120.2(6)$ & 120.085 \\
\hline$C(15)-C(16)-C(17)$ & $121.1(6)$ & 120.594 \\
\hline
\end{tabular}




\begin{tabular}{lll}
\hline $\mathrm{C}(18)-\mathrm{C}(17)-\mathrm{C}(16)$ & $119.4(6)$ & 119.381 \\
$\mathrm{C}(17)-\mathrm{C}(18)-\mathrm{C}(19)$ & $119.7(5)$ & 120.426 \\
$\mathrm{C}(17)-\mathrm{C}(18)-\mathrm{C}(21)$ & $118.6(5)$ & 119.363 \\
$\mathrm{C}(19)-\mathrm{C}(18)-\mathrm{C}(21)$ & $121.7(5)$ & 120.210 \\
$\mathrm{C}(20)-\mathrm{C}(19)-\mathrm{C}(18)$ & $120.1(6)$ & 119.418 \\
$\mathrm{C}(19)-\mathrm{C}(20)-\mathrm{C}(15)$ & $120.9(6)$ & 120.549 \\
$\mathrm{~N}(5)-\mathrm{C}(21)-\mathrm{C}(18)$ & $179.0(7)$ & 178.913 \\
\hline
\end{tabular}


Table S5: Comparison of bond lengths in the moieties of anion and cation between single crystal at $296 \mathrm{~K}$ and geometry optimization in $\beta-[\mathrm{CN}-\mathrm{Py}]\left[\mathrm{Ni}(\mathrm{mnt})_{2}\right]$

\begin{tabular}{|c|c|c|}
\hline \multirow{2}{*}{ Atom pair } & \multicolumn{2}{|c|}{ Bond distance / $\AA$} \\
\hline & Single crystal daata & Geometry optimization \\
\hline Ni1...S3 & $2.1374(8)$ & 2.150 \\
\hline Ni1...S1 & $2.1390(8)$ & 2.139 \\
\hline Ni1...S4 & $2.1427(8)$ & 2.149 \\
\hline Ni1...S2 & $2.1501(8)$ & 2.156 \\
\hline S1...C6 & $1.711(3)$ & 1.715 \\
\hline $\mathrm{S} 2 \ldots \mathrm{C} 7$ & $1.724(3)$ & 1.720 \\
\hline $\mathrm{S} 3 \ldots \mathrm{C} 2$ & $1.706(3)$ & 1.722 \\
\hline $\mathrm{S} 4 \ldots \mathrm{C} 3$ & $1.718(3)$ & 1.720 \\
\hline $\mathrm{N} 1 \ldots \mathrm{C} 1$ & $1.137(4)$ & 1.185 \\
\hline $\mathrm{N} 2 \ldots \mathrm{C} 4$ & $1.140(4)$ & 1.186 \\
\hline $\mathrm{N} 3 \ldots \mathrm{C} 5$ & $1.141(4)$ & 1.185 \\
\hline $\mathrm{N} 4 \ldots \mathrm{C} 8$ & $1.146(4)$ & 1.185 \\
\hline $\mathrm{N} 5 \ldots \mathrm{C} 21$ & $1.123(4)$ & 1.182 \\
\hline N6...C9 & $1.330(4)$ & 1.356 \\
\hline N6...C13 & $1.330(3)$ & 1.355 \\
\hline N6...C14 & $1.487(3)$ & 1.487 \\
\hline $\mathrm{C} 1 \ldots \mathrm{C} 2$ & $1.439(4)$ & 1.407 \\
\hline $\mathrm{C} 2 \ldots \mathrm{C} 3$ & $1.356(4)$ & 1.386 \\
\hline $\mathrm{C} 3 \ldots \mathrm{C} 4$ & $1.426(4)$ & 1.407 \\
\hline $\mathrm{C} 5 \ldots \mathrm{C} 6$ & $1.423(4)$ & 1.411 \\
\hline $\mathrm{C} 6 \ldots \mathrm{C} 7$ & $1.359(4)$ & 1.394 \\
\hline $\mathrm{C} 7 \ldots \mathrm{C} 8$ & $1.428(4)$ & 1.410 \\
\hline $\mathrm{C} 9 \ldots \mathrm{C} 10$ & $1.355(4)$ & 1.379 \\
\hline $\mathrm{C} 10 \ldots \mathrm{C} 11$ & $1.363(5)$ & 1.393 \\
\hline $\mathrm{C} 11 \ldots \mathrm{C} 12$ & $1.367(5)$ & 1.391 \\
\hline $\mathrm{C} 12 \ldots \mathrm{C} 13$ & $1.353(4)$ & 1.382 \\
\hline $\mathrm{C} 14 \ldots \mathrm{C} 15$ & $1.510(4)$ & 1.510 \\
\hline $\mathrm{C} 15 \ldots \mathrm{C} 16$ & $1.369(4)$ & 1.398 \\
\hline $\mathrm{C} 15 \ldots \mathrm{C} 20$ & $1.378(4)$ & 1.398 \\
\hline $\mathrm{C} 16 \ldots \mathrm{C} 17$ & $1.375(4)$ & 1.388 \\
\hline $\mathrm{C} 17 \ldots \mathrm{C} 18$ & $1.365(4)$ & 1.403 \\
\hline C18...C19 & $1.367(4)$ & 1.403 \\
\hline $\mathrm{C} 18 \ldots \mathrm{C} 21$ & $1.450(5)$ & 1.420 \\
\hline C19...C20 & $1.374(4)$ & 1.387 \\
\hline
\end{tabular}


Table S6: Comparison of bond angles in the moieties of anion and cation between single crystal at $296 \mathrm{~K}$ and geometry optimization in $\beta-[\mathrm{CN}-\mathrm{Py}]\left[\mathrm{Ni}(\mathrm{mnt})_{2}\right]$

\begin{tabular}{|c|c|c|}
\hline \multirow{2}{*}{$\angle$ atom1-atom2-atom3 } & \multicolumn{2}{|c|}{ Bond angles $/^{\circ}$} \\
\hline & Single crystal daata & Geometry optimization \\
\hline $\mathrm{S}(3)-\mathrm{Ni}(1)-\mathrm{S}(1)$ & $86.36(3)$ & 86.623 \\
\hline $\mathrm{S}(3)-\mathrm{Ni}(1)-\mathrm{S}(4)$ & $92.04(3)$ & 91.743 \\
\hline $\mathrm{S}(1)-\mathrm{Ni}(1)-\mathrm{S}(4)$ & $178.28(3)$ & 178.363 \\
\hline $\mathrm{S}(3)-\mathrm{Ni}(1)-\mathrm{S}(2)$ & $176.75(3)$ & 175.768 \\
\hline $\mathrm{S}(1)-\mathrm{Ni}(1)-\mathrm{S}(2)$ & $92.29(3)$ & 92.010 \\
\hline $\mathrm{S}(4)-\mathrm{Ni}(1)-\mathrm{S}(2)$ & $89.28(3)$ & 89.613 \\
\hline $\mathrm{C}(6)-\mathrm{S}(1)-\mathrm{Ni}(1)$ & $103.97(10)$ & 104.851 \\
\hline $\mathrm{C}(7)-\mathrm{S}(2)-\mathrm{Ni}(1)$ & $102.93(10)$ & 103.875 \\
\hline $\mathrm{C}(2)-\mathrm{S}(3)-\mathrm{Ni}(1)$ & $103.82(10)$ & 104.540 \\
\hline $\mathrm{C}(3)-\mathrm{S}(4)-\mathrm{Ni}(1)$ & $103.64(10)$ & 104.488 \\
\hline $\mathrm{C}(9)-\mathrm{N}(6)-\mathrm{C}(13)$ & $120.3(3)$ & 121.053 \\
\hline $\mathrm{C}(9)-\mathrm{N}(6)-\mathrm{C}(14)$ & $119.7(3)$ & 119.141 \\
\hline $\mathrm{C}(13)-\mathrm{N}(6)-\mathrm{C}(14)$ & $120.0(3)$ & 119.803 \\
\hline $\mathrm{N}(1)-\mathrm{C}(1)-\mathrm{C}(2)$ & $177.7(3)$ & 176.013 \\
\hline$C(3)-C(2)-C(1)$ & $122.1(3)$ & 124.282 \\
\hline $\mathrm{C}(3)-\mathrm{C}(2)-\mathrm{S}(3)$ & $120.6(2)$ & 119.442 \\
\hline $\mathrm{C}(1)-\mathrm{C}(2)-\mathrm{S}(3)$ & $117.3(2)$ & 116.271 \\
\hline $\mathrm{C}(2)-\mathrm{C}(3)-\mathrm{C}(4)$ & $122.8(3)$ & 123.604 \\
\hline$C(2)-C(3)-S(4)$ & $119.9(2)$ & 119.745 \\
\hline $\mathrm{C}(4)-\mathrm{C}(3)-\mathrm{S}(4)$ & $117.3(2)$ & 116.639 \\
\hline $\mathrm{N}(2)-\mathrm{C}(4)-\mathrm{C}(3)$ & 179.2(3) & 176.861 \\
\hline $\mathrm{N}(3)-\mathrm{C}(5)-\mathrm{C}(6)$ & $176.2(3)$ & 176.076 \\
\hline$C(7)-C(6)-C(5)$ & $123.2(3)$ & 123.907 \\
\hline$C(7)-C(6)-S(1)$ & $119.9(2)$ & 119.189 \\
\hline $\mathrm{C}(5)-\mathrm{C}(6)-\mathrm{S}(1)$ & $116.8(2)$ & 116.809 \\
\hline$C(6)-C(7)-C(8)$ & $122.1(3)$ & 121.526 \\
\hline$C(6)-C(7)-S(2)$ & $120.7(2)$ & 119.949 \\
\hline $\mathrm{C}(8)-\mathrm{C}(7)-\mathrm{S}(2)$ & $117.2(2)$ & 118.469 \\
\hline $\mathrm{N}(4)-\mathrm{C}(8)-\mathrm{C}(7)$ & $178.1(4)$ & 178.324 \\
\hline $\mathrm{N}(6)-\mathrm{C}(9)-\mathrm{C}(10)$ & $120.8(3)$ & 120.548 \\
\hline$C(9)-C(10)-C(11)$ & 119.3(3) & 119.213 \\
\hline$C(10)-C(11)-C(12)$ & $119.5(3)$ & 119.471 \\
\hline $\mathrm{C}(13)-\mathrm{C}(12)-\mathrm{C}(11)$ & 119.1(3) & 119.480 \\
\hline $\mathrm{N}(6)-\mathrm{C}(13)-\mathrm{C}(12)$ & $121.0(3)$ & 120.225 \\
\hline $\mathrm{N}(6)-\mathrm{C}(14)-\mathrm{C}(15)$ & $112.3(2)$ & 112.217 \\
\hline$C(16)-C(15)-C(20)$ & $118.6(3)$ & 119.074 \\
\hline$C(16)-C(15)-C(14)$ & $120.9(3)$ & 120.407 \\
\hline$C(20)-C(15)-C(14)$ & $120.5(3)$ & 120.487 \\
\hline$C(15)-C(16)-C(17)$ & $120.9(3)$ & 120.955 \\
\hline
\end{tabular}




\begin{tabular}{lll}
\hline $\mathrm{C}(18)-\mathrm{C}(17)-\mathrm{C}(16)$ & $119.9(3)$ & 119.449 \\
$\mathrm{C}(17)-\mathrm{C}(18)-\mathrm{C}(19)$ & $119.8(3)$ & 120.021 \\
$\mathrm{C}(17)-\mathrm{C}(18)-\mathrm{C}(21)$ & $120.2(3)$ & 120.212 \\
$\mathrm{C}(19)-\mathrm{C}(18)-\mathrm{C}(21)$ & $119.9(3)$ & 119.744 \\
$\mathrm{C}(18)-\mathrm{C}(19)-\mathrm{C}(20)$ & $120.1(3)$ & 119.732 \\
$\mathrm{C}(19)-\mathrm{C}(20)-\mathrm{C}(15)$ & $120.6(3)$ & 120.744 \\
$\mathrm{~N}(5)-\mathrm{C}(21)-\mathrm{C}(18)$ & $179.5(4)$ & 179.344 \\
\hline
\end{tabular}

\section{References}

2. Xie, J. L.; Ren, X. M.; He, C.; Song, Y.; Meng, Q. J.; Kremer, R. K.; Yao, Y. G. Chem. Phys. Lett. 2003, 369, 41-48. 